\title{
Polypharmacy in Geriatrics
}

\author{
Rajesh Chauhan*, Shruti Chauhan and Ajay Kumar Singh
}

Family Healthcare Centre, 154 Sector 6-B (HIG), AvasVikas Colony, Sikandra, Agra, India

\section{Editorial}

Polypharmacy yet has no globally accepted single definition. Nevertheless, we all know that polypharmacy can at times turn out to be adverse for patients of all ages. However, in the elderly geriatric population there are more chances of adverse effects when the intrinsic correcting and compensatory mechanisms are already getting compromised by aging process. Nonetheless consideration of polypharmacy seems to be guided by circumstances, individual preferences, personal justifications, notions, ideas, concepts, and the 'trend'. Differentiation is therefore needed. Differentiation is needed between the required, essential, and justified polypharmacy, and the one that which is unwarranted, not required and can have adverse consequences.

Therefore, first and the foremost thing that needs to be done is to define polypharmacy within reasonable limits, and this will have to be on an individualized basis and as required for the effective management of all the morbidities requiring intervention. Need based prescriptions should become an accepted concept. Concurrent use of homeopathic, ayurvedic, herbal, Unani medicines, local medicines like Chinese, Tibetan and 'Traditional Medicines', etc, should be considered falling within the domain of polypharmacy, especially if their pharmacopeia and pharmaceutical properties are not understood by the treating physician, so as to be leaving many lacunae in the understanding of their usefulness, interactions, and their adverse effects. More importantly, it needs to be ascertained that using modern medicine (allopathic) along with any of these medicines do not interact, potentiate or have any adverse effect on the elderly patients.

Some of the researchers do take into consideration the multivitamins while some won't, despite enough evidence as excess buildup within a body of water insoluble vitamins may cause problems. Personal fads, concurrent use of alcohol and substance abuse may also play up and complicate the issue. Therefore, it is essential that once a thorough clinical and a detailed history has been taken, it will be the time to go through the treatment history. Changing things will require taking an extra step and some extra effort. In that check, yourself all the different medicines your patient might be using, and be sure what is now needed by your patient, and what is not. Give a chance to your patients or their care givers to come and show all the medicines that are being used regularly, intermittently, or occasionally. Listen to your patient (and their care givers), and give him or her time to tell you why he or she has been using all of those drugs, lotions, ointments, elexirs, medicines that could have been prescribed long time ago, or procured OTC (over the counter).

Multimorbidities will continue to weigh down some of the susceptible geriatric population, and for sure they will require different medicines and their combinations as well. There will be a need to step into your patient's shoes and see from his or her angle as well. Try to get to the bottom whether the intake of non-prescribed drugs is by mistake or ignorance, or is it by considered opinion coming from family, friends, other patients with similar problems, or through a 'friendly advice' by a pharmacist, nurse, or any other clinic or hospital staff; whatever. Try getting out the real intent and purpose of using a particular drug or medicine, when not advised by his or her doctor. Is it the result of any associated difficulty in comprehension, language, or possibly memory or vision problem? Or is the problem iatrogenic? Illegible writing, overwriting, typo errors, etc, are some common errors, as is the provision of inadequate information about discontinuation or reduction of some medicine. A prescription that was intended and meant for a specific duration only, may get repeated inadvertently, which would be add to the problem of polypharmacy. In some cases a home-visit can be a good idea to investigate and get a firsthand knowledge of the drugs and medicines/supplements that are kept handy for regular or occasional use by the patients.

Once the extra medicines, supplements, etc, have been identified, weaning them away would be the next step. Weaning away the unwanted medicines and supplements can be difficult, and must be done with all care and expertise at the command, with proper explanations and justifications for the need of their removal or weaning away. At times, some patients may be so much psychologically 'attached' to some or the other medicine or supplement, and to make them let go may be a tricky job.

It could be worthwhile reminding your patients the A, B, C, and D in order to rein in polypharmacy:

\section{AVOID fancy and non-prescription drugs.}

BEWARE of unknown problems due to concomitant use of homeopathic, ayurvedic, herbal, Unani medicines, and local medicines like Chinese, Tibetan and 'Traditional Medicines', 'supplements', alcohol and substance abuse, etc.

COUNT the number of your pills, and take each of them only if necessary, and upon.

DOCTOR's orders only.

${ }^{*}$ Corresponding author: Rajesh Chauhan, Family Healthcare Centre, 154 Sector 6-B (HIG), AvasVikas Colony, Sikandra, Agra, India, Tel: 9197600211912; E-mail: drchauhanrajesh@yahoo.com

Received September 19, 2017; Accepted September 19, 2017; Published September 25, 2017

Citation: Chauhan R, Chauhan S, Singh AK (2017) Polypharmacy in Geriatrics. J Gerontol Geriatr Res 6: e147. doi:10.4172/2167-7182.1000e147

Copyright: (c 2017 Chauhan R, et al. This is an open-access article distributed under the terms of the Creative Commons Attribution License, which permits unrestricted use, distribution, and reproduction in any medium, provided the original author and source are credited. 